\title{
José Cruz
}

+ Hernán Cruz + Ana Turell + Juan Purcell (asoc. UAI)

Dos obras, horizontales y paralelas a la longitud del territorio chileno, dan cuenta de maneras distintas de situarse entre el valle central y la cordillera. Con maderas, una, y con una arquitectura blanca, la otra, apelan a la levedad y al arraigo respectivamente, y mediante la abstracción, median entre las múltiples dimensiones del acontecer de una vida en el interior, y la extensión como dimensión territorial del quebacer arquitectónico.

Two works, horizontal and parallel with Chile's longitude, offer different ways of taking position between the Central Valley and the cordillera. One with wood, the other with simple architecture, they call on lightness and rootedness respectively, and in abstract measure the many dimensions of a life-in-progress in the interior, and extension as a territorial dimension of the architectonic task.

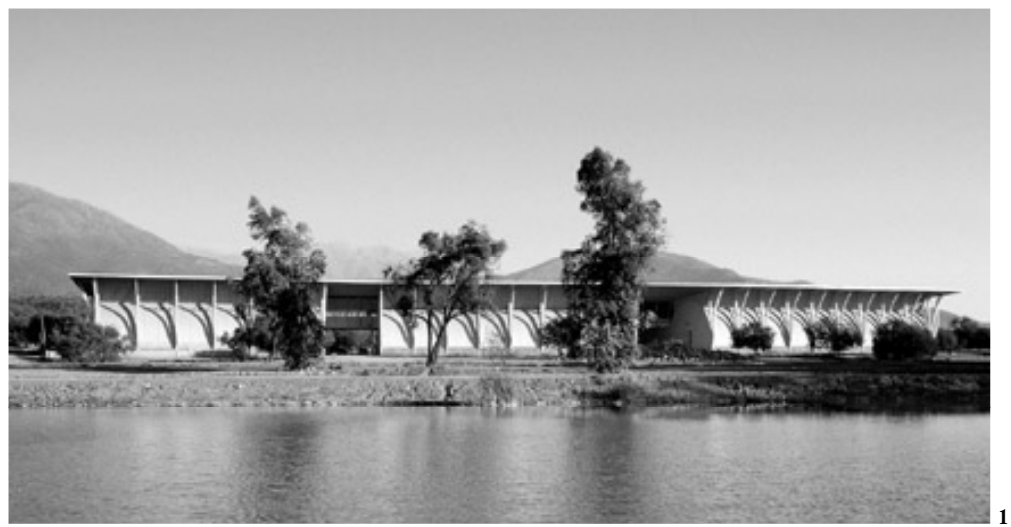

\section{Bodegas de Vinificación Viña Pérez Cruz}


Estas bodegas se sitúan próximas a los viñedos, en el fundo Liguai de Huelquén, Paine, sobre la franja en que el valle se encuentra con los primeros faldeos de la cordillera. Desde el lugar aparece lo extenso del valle cultivado y la naturaleza agreste de los faldeos y los cerros, hasta las cumbres -que parte del año permanecen cubiertas de nieve-, haciéndose presente esa dimensión rural propia de un campo con tamaño y entidad de fundo y no meramente de terrenos para el cultivo. Un campo que en sus 530 hectáreas, además de las viñas, otorga espacio a la cría de caballos chilenos y otras faenas, dentro de esa unidad con la que un fundo puede regentar lo diverso, mediante aquella libertad que sabe cuidar, incluso, ese resto de breve indolencia como parte de su propio desconocido. Tal cosa nos permitió concebir un primer tamaño que no vacila en celebrar su propia existencia para recoger esas dimensiones como aquello que acompaña de suyo el cultivo de la uva, para que estas bodegas respondan al modo como se habita el campo y, al igual que el vino, puedan dejar colocada la innovación dentro de una cierta tradición. Pero se trata que estas dimensiones no sean incluidas desde su condición literal y narrativa, sino pensadas a partir de una elaboración abstracta, que considera cinco coordenadas.

I. Para una obra de arquitectura emplazarse en el campo significa quedar orientada sin la jerarquía de un delante y un detrás como en la ciudad. Esto hace pensar en un edificio con un perímetro habitable, sin revés ni derecho, equivalente en todos sus frentes, capaz de crear una transición entre el interior y el exterior. Se propone desplegar este perímetro como un borde sombreado y protegido de la lluvia, conformando un espesor, matizado por el claroscuro de las sombras y la luz.

II. La intemperie del campo, con su situación de cielo abierto, muestra la importancia que posee una sombra como primera instancia para hacer habitable el espacio: muchas faenas del campo tan sólo requieren de ésta para realizarse. Ello nos lleva a concebir primeramente el tamaño de una gran sombra bajo la cual puede espaciarse el interior. Tal cosa supone una separación entre la superficie de la cubierta, que arroja esa sombra, y el límite del vacío interno -conformado esta vez por la continuidad de las bóvedas-, abriéndose entre ambas una profundidad vertical que se hace visible como espesor de luz descendente hacia el interior, y que se construye mediante una estructura que, manteniendo la distancia de esta separación, reúne en un solo orden la geometría curva de las bóvedas con la geometría recta de los planos de la cubierta, para crear ese amplio intersticio por donde el aire puede circular para ventilar de modo natural las naves.

III. Se propone que la obra despierte, como el vino, cierta voluptuosidad de los sentidos, encarnando la dimensión sensible de la forma mediante la relación entre la materia y el espacio. En su despliegue con la luz, la madera otorga esa multiplicidad de matices que, como el aroma, el sabor y el color del vino, son concertados en la obra para construir la duración por medio de una diversidad de tamaños que conforman los espesores y profundidades que nos deja ante y dentro de aquello que busca no agotarse en una primera mirada, sino poder prolongarse hacia una segunda instancia que reciba las dimensiones de esa heterogénea unidad puestas en juego en la obra ${ }^{1}$.

IV. Este proceso de elaboración del vino, se lleva a cabo con operaciones paralelas, en simultaneidad, y operaciones sucesivas, en secuencialidad. El edificio recoge la simultaneidad con el paralelismo -naves paralelas-y la secuencia mediante la longitud. Tres naves dobles que se enlazan entre sí mediante dos pórticos, o patios cubiertos -utilizados como patios de vendimiapor medio de los cuales se articula el giro del edificio.

V. Una parte importante del trabajo de una bodega se realiza a una cierta altura del suelo: el nivel que establecen las bocas de las cubas de fermentación; esta altura se incorpora a la obra formando un corredor central elevado del suelo y alojado entre ambas bóvedas, una espina dorsal continua por la que discurren las instalaciones y por la que es posible desplazarse de extremo a extremo, sin perder el paso, a lo largo de los $140 \mathrm{~m}, \mathrm{y}$ a una altura que permite acceder a las pasarelas de inspección de las cubas, a la losa mesanina y al área de laboratorios.

La madera entrega las condiciones térmicas adecuadas y la tactilidad necesaria para que la masa edificada alcance una vibración que otorgue levedad a este tamaño.

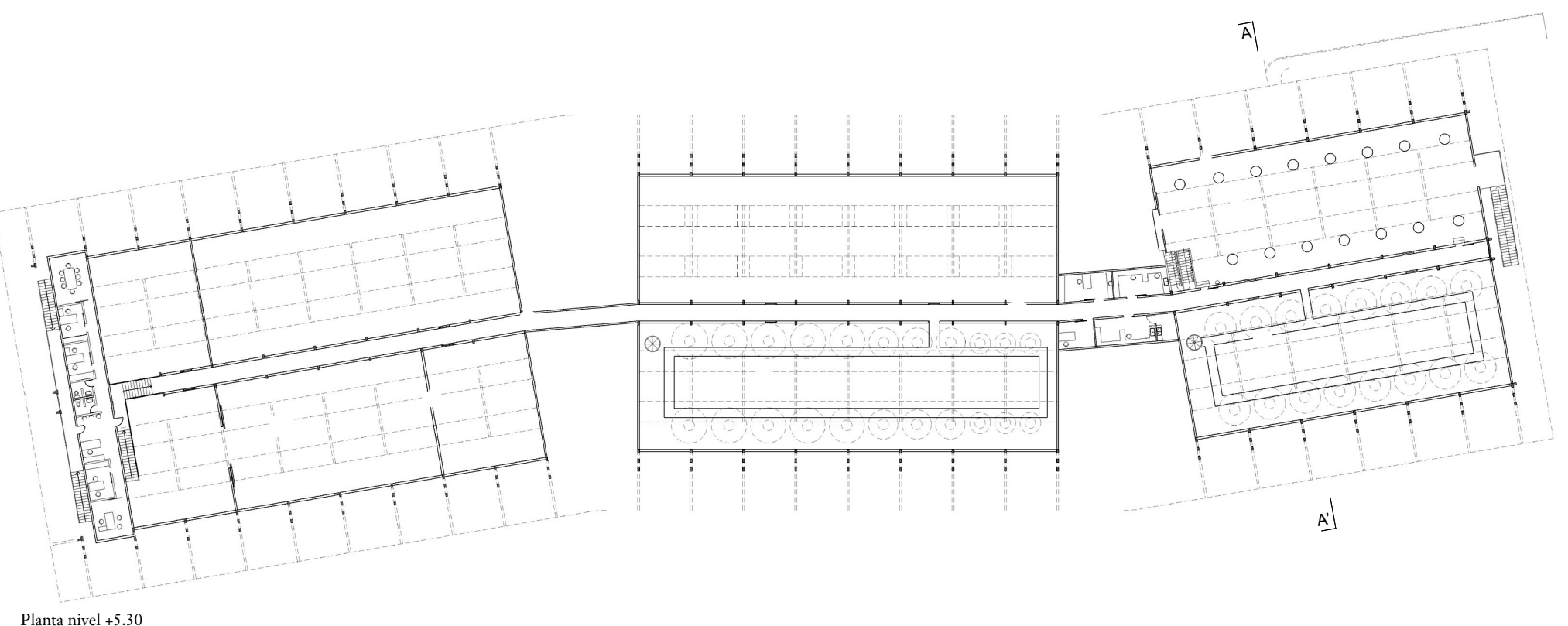

Planta nivel +5.30

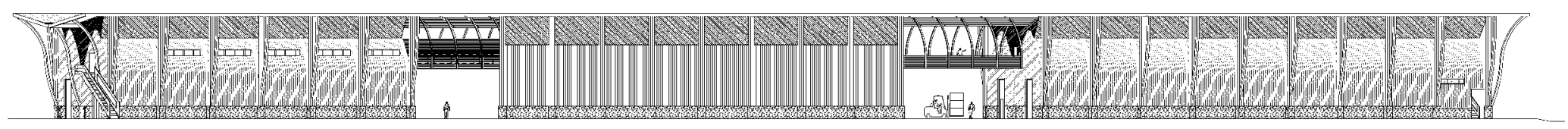

Elevación longitudinal 


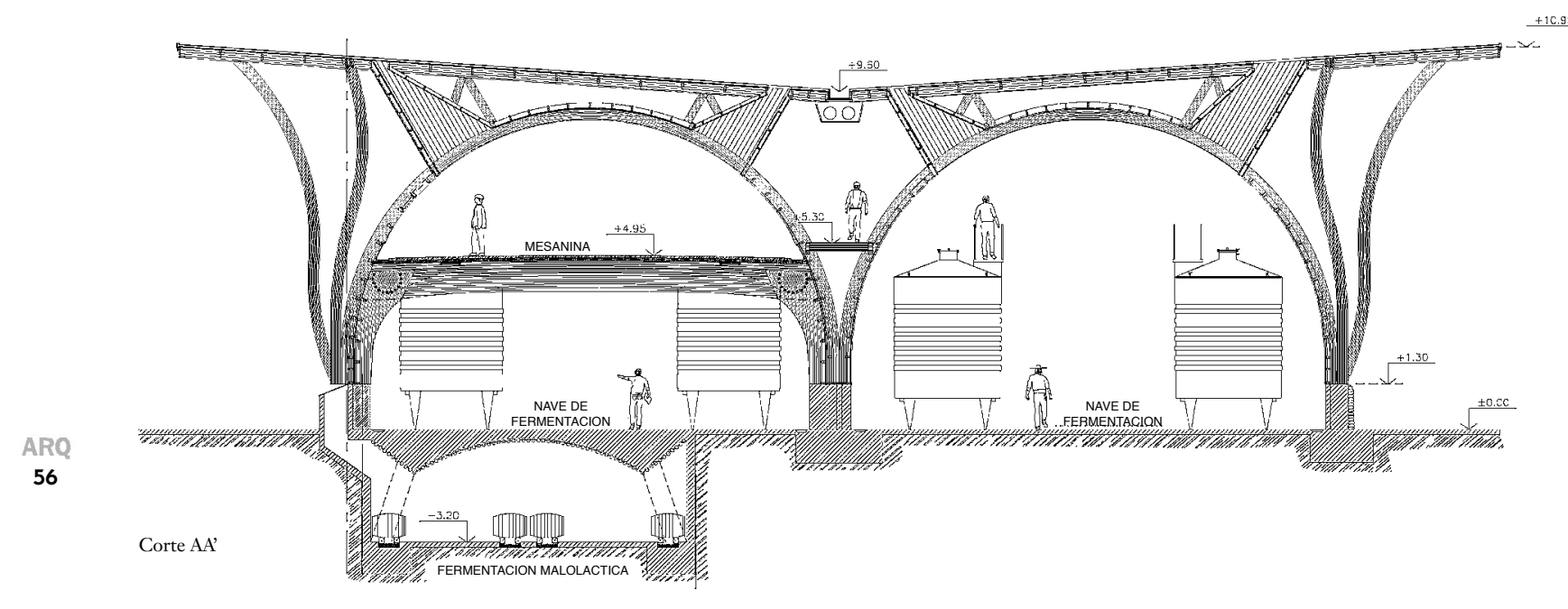

2 Vista desde puentes de inspección de nave de

3 Nave de fermentación

maloláctica (subterránea)

4 Cielo nave de fermentación

5 Fachada nor-poniente

6 Patio de descarga

7 Detalle desde patio de

recepción de vendimia
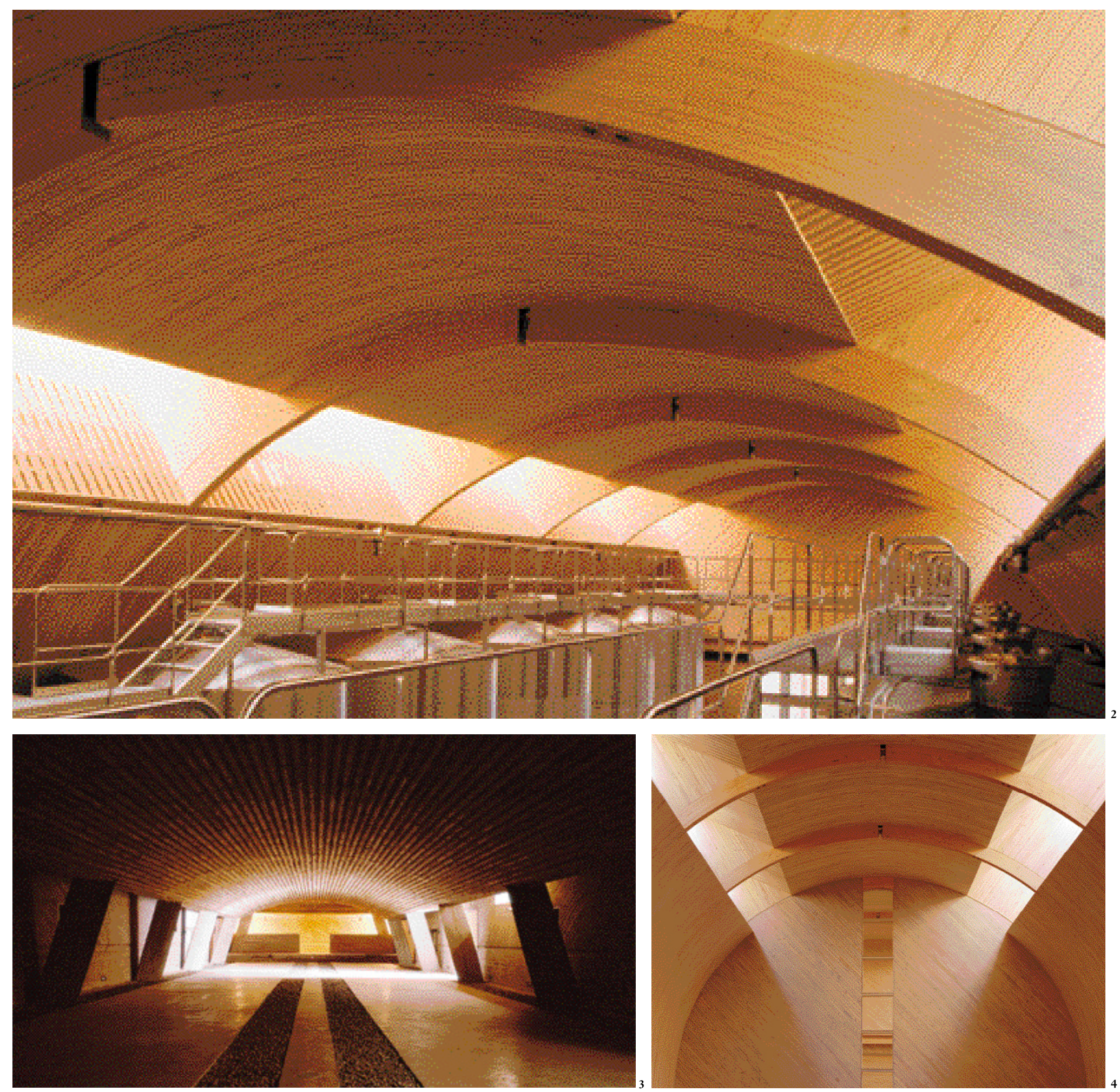
Volvieubo soste las obsersaciaines del campor I de la vegetación mativa dibyjeda en el lugar: los apsetadon namajes que deade de suclo se ebren hacie la alto nefendo atrapa. do el spreior, el aire entre ells - Tal espesos.
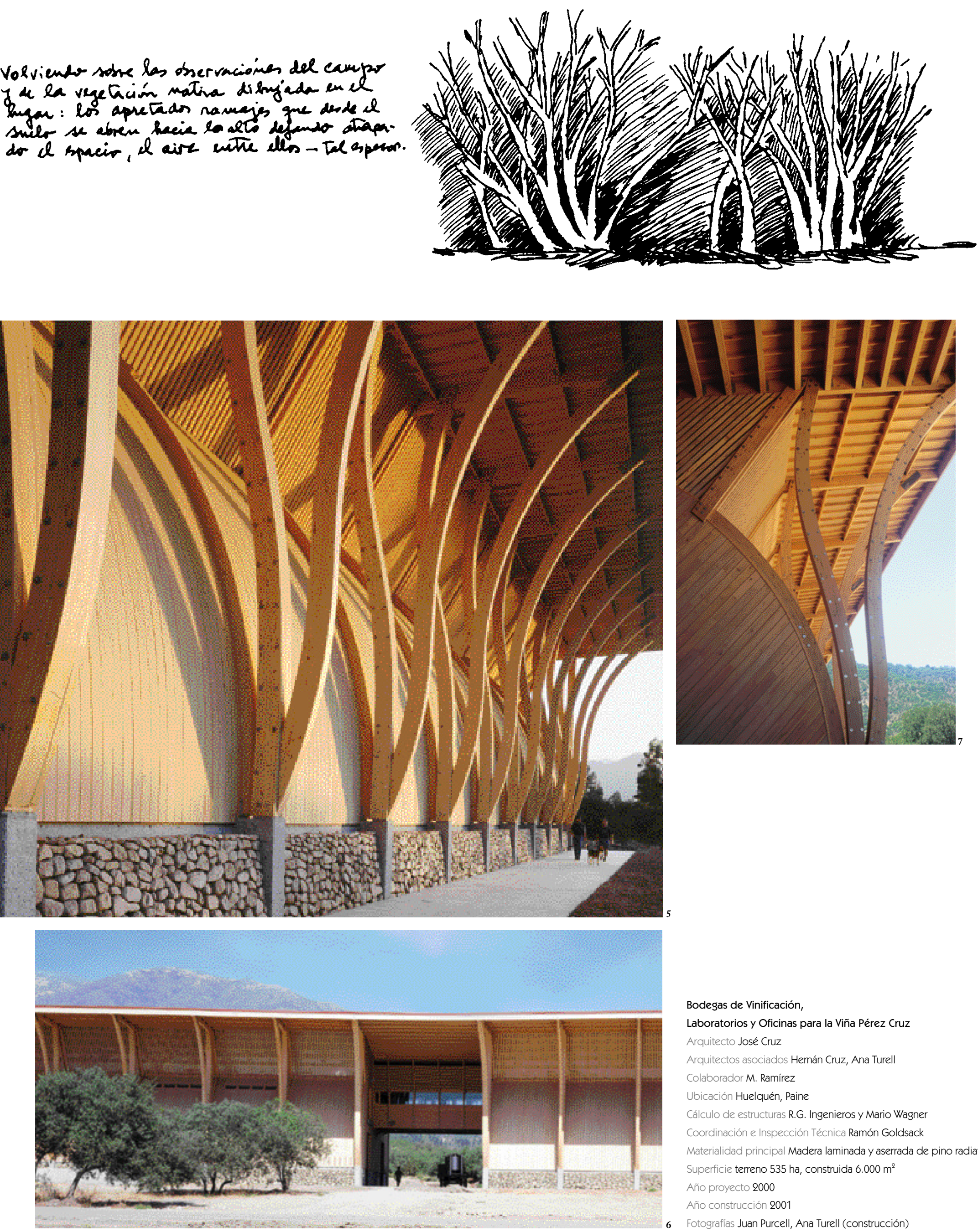

Bodegas de Vinificación

Laboratorios y Oficinas para la Viña Pérez Cruz

Arquitecto José Cruz

Arquitectos asociados Hernán Cruz, Ana Turell

Colaborador M. Ramírez

Ubicación Huelquén, Paine

Cálculo de estructuras R.G. Ingenieros y Mario Wagner

Coordinación e Inspección Técnica Ramón Goldsack

Materialidad principal Madera laminada y aserrada de pino radiata

Superficie terreno 535 ha, construida $6.000 \mathrm{~m}^{2}$

Año proyecto 2000

Año construcción 2001

Fotografías Juan Purcell, Ana Turell (construcción) 

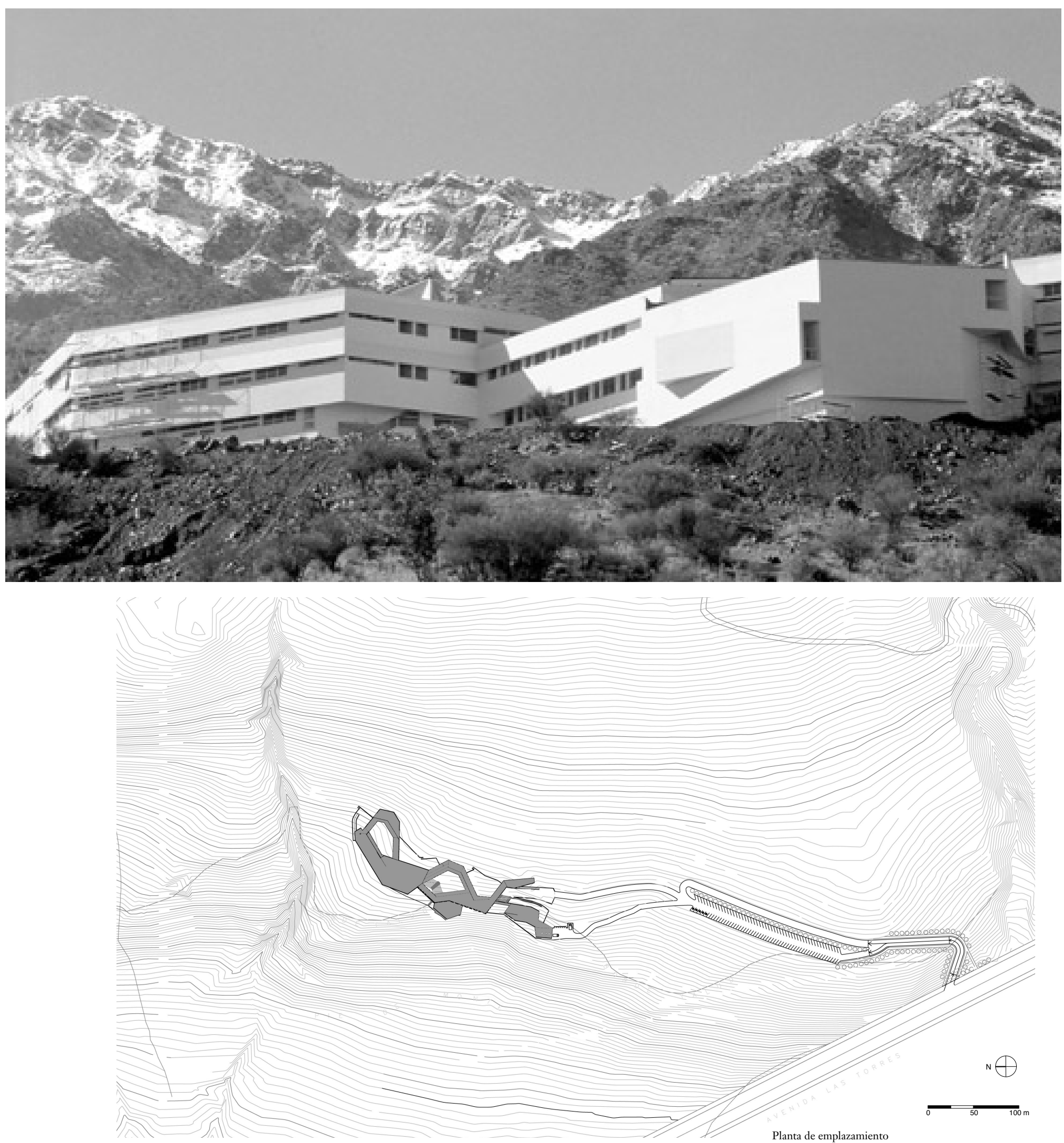
La Universidad se concibe a partir de cinco coordenadas simultáneas, como los cinco vértices de un pentágono, equivalentes, en cuanto imprescindibles, para construirlo.

\section{Ubicación}

La Universidad se sitúa en un lugar singular, aquel en el cual la ciudad discurre su realidad actual: el borde. Esto supone un primer intento por cavilar precisamente aquello que pueda ser hoy la universidad. A diferencia de emplazarse en el centro que podría indicar la continuación de lo ya realizado.

Un borde que es también un límite: aquel entre la ciudad y la naturaleza; conformación geográfica y frontera de nuestro país: la cordillera de los Andes con su dimensión continental. Este hecho constituye la primera afirmación arquitectónica de la universidad, cual es esta ubicación única que la sitúa en una dimensión americana: emplazarse crucialmente ante la vastedad, lo cual otorga: -Esta magnitud propia del país y del continente: La extensión

- La relación ciudad - naturaleza: (una disputa del mundo actual)

-La altitud y la pendiente con sus relaciones del arriba y abajo propias de los cerros

-Una doble dimensión de lejanía: hacia el valle y la ciudad hacia la cordillera y la vastedad de la naturaleza El lugar por su tamaño y ubicación permite detenerse libre del tráfago de la ciudad pero sin abandonarla. Sin estar en medio, tampoco se queda fuera ya que se permanece ante. Ante la ciudad, sobre este borde natural; una altitud que permite contemplarla con aquella distancia que la reúne en una visión de totalidad, formando parte de un horizonte de lejanía, equidistante entre la ciudad y la naturaleza, entre el valle y la vastedad del macizo cordillerano, con ese doble horizonte de la altura y de la amplitud.

Desde este lugar es posible, al inicio de la jornada, ver emerger los rayos de un primer sol colándose entre lo alto de las cumbres, y contemplar su ocaso sobre la ciudad, abriendo la profundidad del valle y su inmensidad. Lugar propicio para que el estudiante pueda allí imaginar su destino.

\section{Totalidad: tamaño y extensión}

Para concebir el tamaño habitable de la obra como una totalidad espaciada desde la extensión, se la piensa como un desprendimiento del cielo abierto, cual si su proyección sobre la tierra, tocara suelo en algunos puntos y en otros fuese detenida a cierta altura para arrojar su sombra. De esta forma unos cuerpos de edificio hacen pie abajo y otros lo hacen más arriba, uniéndose por el aire, mediante tramos suspendidos que, atravesando sobre los patios, construyen su profundidad y otorgan sombra y protección de la lluvia.

Un modo de espaciar la arquitectura mediante la extensión, que enlazando un ritmo de explanadas, patios y anfiteatros, anuda el arriba y el abajo, el suelo con el cielo - propios de un lugar en pendiente-, mediante estos tramos suspendidos que le dan medida a ese cielo abierto, proyectándolo sobre el suelo. Así, lo singular de este lugar se vuelve una presencia y no mera representación (ver coordenada III).

Es necesario observar que desde lo situado en esa altitud en la que se emplaza la obra, sobre los faldeos cordilleranos, la potencia de lejanía entre el horizonte del valle y el de los cerros es diversa: hacia el valle el espacio se desboca, fugándose, en cambio hacia la cordillera se retiene. Para que esta doble dimensión de lejanía, pueda construirse con equivalencia, se tiene lo siguiente:
En los exteriores, la fuga se gobierna mediante el atravieso de estos cuerpos suspendidos, por sobre los anfiteatros y graderías que descienden desde los patios en dirección al valle -permitiendo al ojo medir la lejanía y graduar la fuga del espacio.

En los recintos interiores, las ventanas orientadas al valle se parapetan tras una suerte de pestañas cual pliegues que descuelgan el propio muro, reteniendo el interior y deteniendo la fuerza de los rayos del sol del poniente en primavera- verano. Del mismo modo, se inventa un interior en el que se habita en consonancia con el lugar $\mathrm{El}$ ascender o descender, se realiza en la continuidad de una pendiente suave-rampas-que incorporan la extensión, como sucede en el cerro, de forma que el ir hacia arriba o hacia abajo se torna en recorrido. Se abandona el carácter "temático" del movimiento vertical de una escalera.

Lo edificado propone lo siguiente:

-patios-atraviesos y no claustros de perímetros encerrados: dejar traspasar la naturaleza

-masas edificadas elevadas: una forma leve de la relación suelo-cerro, suelo-edificio

-edificios que circundan sus vacíos: los patios suspendidos equivalencia de vacío de cielo con vacío de suelo.

-continuidad libre de las fachadas que expanden sus aristas: no al cubo ni a la torre.

\section{Representación y Presencia}

En el mundo antiguo el símbolo tenía capacidad para representar el total.

El saber del hombre se apoyaba en lo simbólico.

Si tomamos un cuadro del renacimiento, por ejemplo,

"La Alegoría de la Primavera" de Botticceli, podemos ver que alude a algo que está fuera, más allá.

El símbolo alcanza aquí la trascendencia mediante la representación de esa otra realidad hacia la que apunta: los antiguos mitos del mundo greco romano. Pero aquí se piensa que el presente de la Universidad no puede ya apoyarse en lo simbólico, pues el mundo ha pasado del símbolo a la función. La voluntad simbólica es hoy nostalgia de un mundo ausente porque carece ya de capacidad para representar una totalidad.

Pero se da un cambio en lo representativo porque la representación dejó de ser simbólica, debido a que el mundo pasó de la trascendencia a la inmanencia. Lo Trascendente no es hoy lo que está fuera, más allá, sino dentro.

En el arte moderno la trascendencia no se alcanza por la representación de otra realidad -mítica o religiosa- sino por la invención de esa realidad que está allí, delante nuestro: la obra.

Es la obra en sí misma la que se vuelve trascendente desde su propia inmanencia. La abstracción nos pone así ante una presencia, no ante una representación.

La arquitectura de esta universidad no se origina desde la representación sino desde la presencia, entendida como aquello que da lugar al acto real de habitar con el cuerpo. Habitar en un equilibrio:

Un equilibrio entre la masa edificada y la extensión natural de los cerros, entregado por la continuidad del tamaño caminable de la universidad. Un equilibrio para que el estar fuera y dentro de las aulas sea habitar el espacio de la universidad.

Un equilibrio que recogiendo las singularidades del lugar, propone para la universidad una espacialidad que trae una plenitud a la contemplación y al estudio, en cuanto hace posible permanecer allí una jornada completa en virtud de la diversidad de espacios que permiten múltiples modos de estar, acogiendo las distintas posturas para el cuerpo.
Esta variación en la diversidad es la que permite permanecer en un lugar sin fatigarse.

Un equilibrio para que la cantidad de alumnos no se vuelva una multitud. Para ello el espacio se piensa desde un recato que no busca el elogio de la muchedumbre, como en esos centros comerciales, que con sus antepechos transparentes hacen visible y -podría decirse- exaltan el gentío en movimiento.

Por el contrario, se trata del uno a uno, para lo cual el espacio desplegado en continuidad es ritmado por la variación, a fin de conformar lugares únicos y no repeticiones homogéneas de un espacio que se extiende hasta alcanzar su tamaño. Por esto mismo los antepechos opacos de las galerías y sus alturas variables cuidan tal recato de la cantidad para no volverla jamás muchedumbre.

\section{Lo Anterior}

La universidad, a diferencia del colegio, descansa lo anterior; un acto anterior a toda tematización. Su ser universidad reposa en un estado a-temático.

(El colegio no necesita esta dimensión porque el niño carece de un anterior a sí mismo. Por ello es reglamentado dentro de una estructura temática en la que incluso los recreos tienen sus juegos tematizados). Se trata de una dimensión no específica -no se está en el laboratorio, ni en la sala de clase, ni en el estar de alumnos. No consiste en descanso, recreo, ni nada organizado. Se trata de lo no específico que debe acoger la universidad para que los alumnos puedan vivir ese momento de lo anterior, para que tal momento tenga esplendor. Si bien los métodos de estudio han cambiado, esto no debería cambiar pues una universidad medita su ser universidad de un modo libre no tematizado. Esto supone una postura frente al "Campus universitario", pues la naturaleza, por sí misma, sin la arquitectura, no puede otorgar una condición que trascienda el estado de recreo, la recreación. Para esto se realiza una invención arquitectónica que introduce la extensión en la forma libre de las circulaciones que se despliegan enlazando galerías, rampas, paseos, patios, graderías, anfiteatros..., conformando un gran interior arquitectónico, que establece un paso gradual entre lo temperado y la intemperie, entre el interior de los edificios y el interior de los patios y recodos. El blanco responde a esta espacialidad desplegada, sin revés ni derecho, desde estos interiores cerrados a los interiores abiertos y a los exteriores, en una continuidad no homogénea, dando cuenta de su tamaño de totalidad concebido como desprendimiento del cielo abierto.

\section{Forma y esplendor de una magnitud}

Corresponde a nuestra cultura occidental que todo lugar dedicado a una actividad u oficio valioso del hombre, vale decir, con un destino mas allá del mero uso, busca no pasar inadvertido, sino alcanzar un esplendor que lo haga visible. En nuestra tradición esto se ha hecho presente en aquellas formas que celebran su existencia. El celebrar supone lo que de algún modo se com-parte; aquello de lo cual se parti-cipa.

Por esto la universidad no se oculta ni mimetiza sino que manifiesta su forma buscando el sentido que corresponde a habitar este lugar. La forma celebra su existencia otorgando esplendor a una de sus magnitudes, en este caso, la de una extensión horizontal desplegada con las variaciones de un ritmo.

Aparece así la arquitectura de la universidad no como un caserío o un villorrio - una serie de pabellones dispersospero tampoco como esas abstracciones universales que podrían estar situadas en cualquier lugar. 

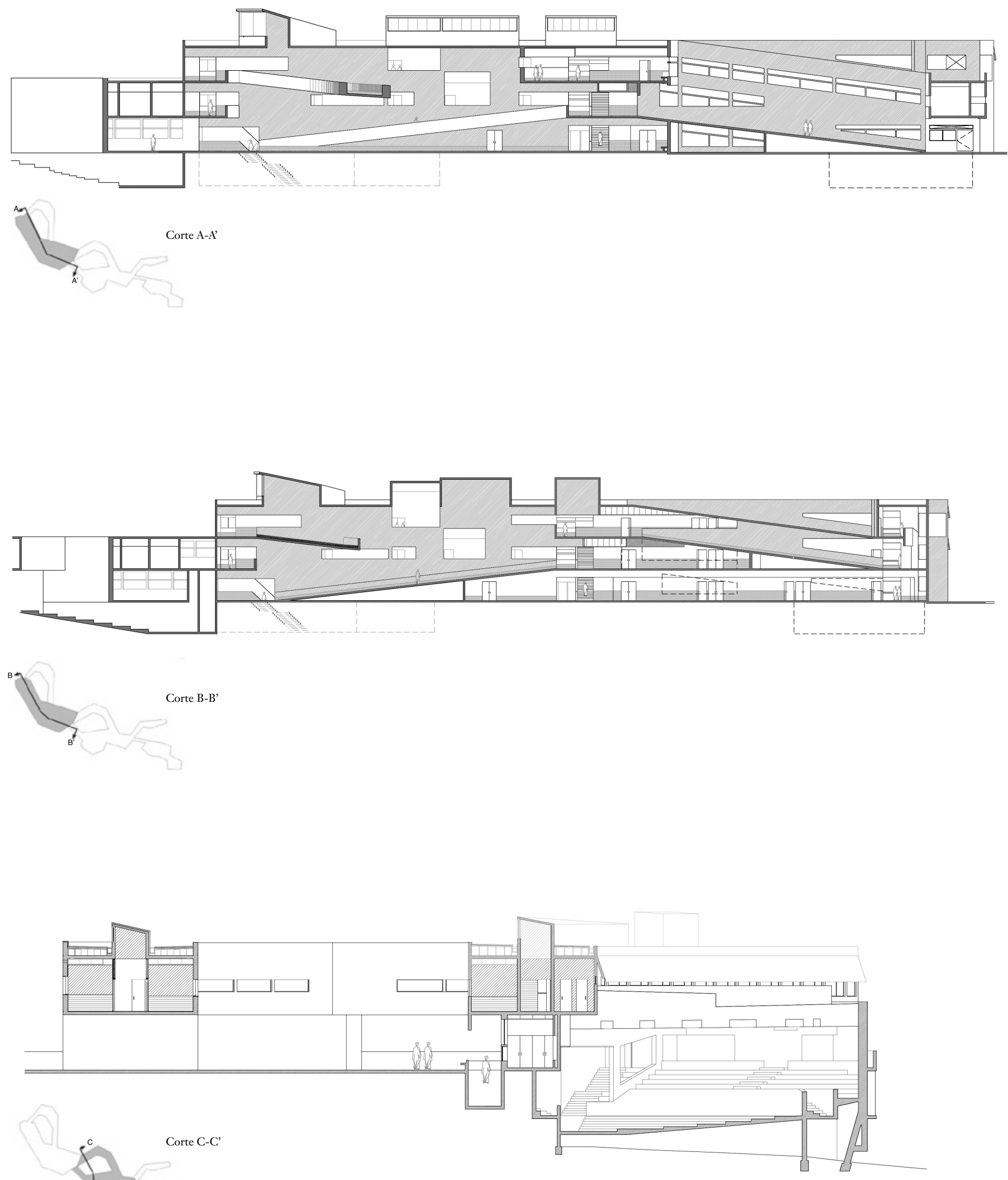
en ln exterios

cono segundo peso la fige del expacio segotierma mediante el atravier de estos cuerpos suspendides, por sobre lon aufiteatros g graderios que desciemdern de los patios en di desciemden de los pation en di valle -
rección al valle -
of medir en lejanitin al la frye del mpen. Proximidad para detener
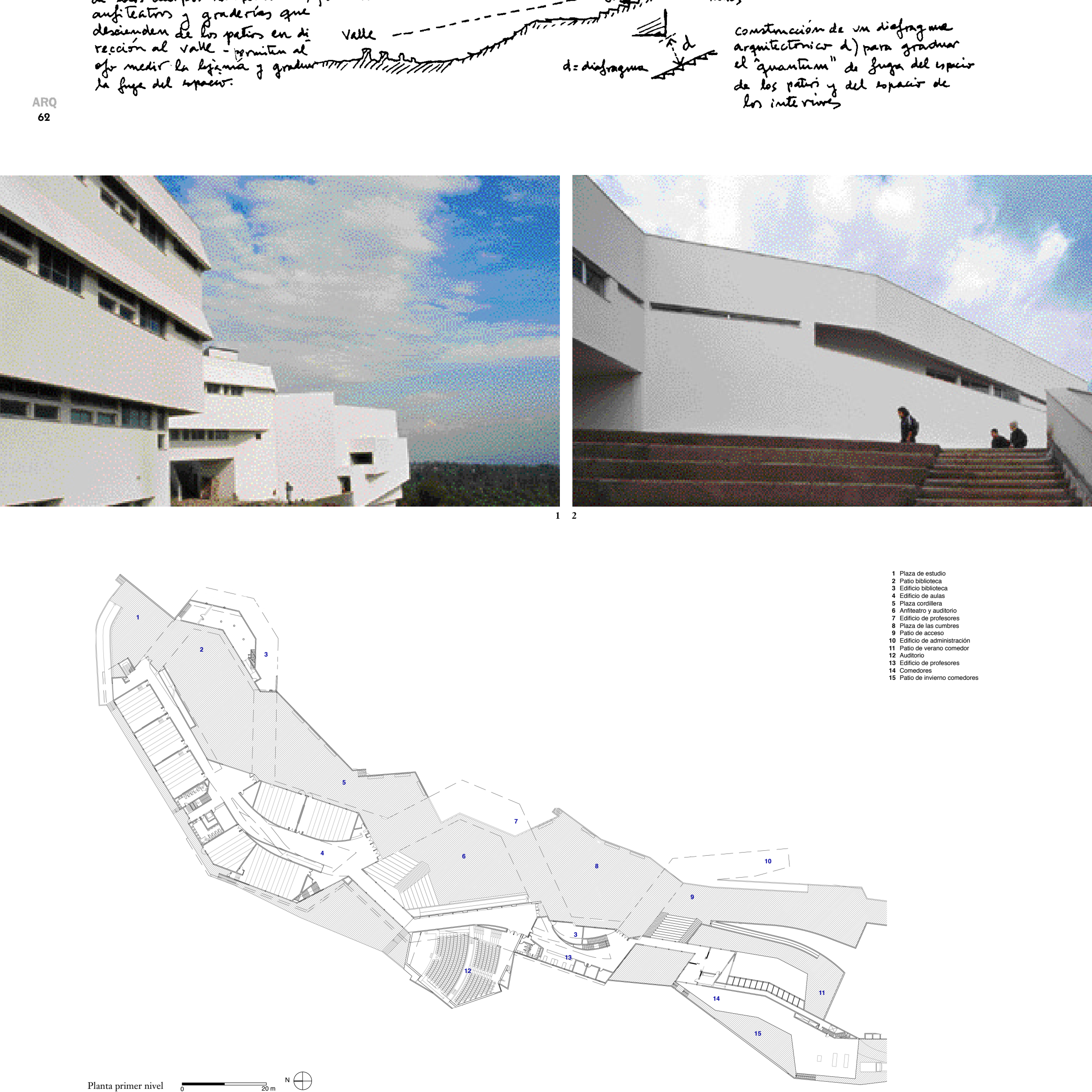
